\title{
The Development and the Use of Experimental Animal Models to Study the Underlying Mechanisms of CA Formation
}

\author{
Tomohiro Aoki' ${ }^{1,2}$ and Masaki Nishimura1 \\ ${ }^{1}$ Department of Neurosurgery, Graduate School of Medicine, Kyoto University, 54 Kawaharacho, Shogoin, Sakyo-ku, \\ Kyoto 606-8501, Japan \\ ${ }^{2}$ Department of Pharmacology, Graduate School of Medicine, Kyoto University, Konoe-cho Yoshida, Sakyo-ku, Kyoto 606-8315, Japan
}

Correspondence should be addressed to Tomohiro Aoki, tomoaoki@kuhp.kyoto-u.ac.jp

Received 24 August 2010; Revised 6 November 2010; Accepted 13 December 2010

Academic Editor: Monica Fedele

Copyright (C) 2011 T. Aoki and M. Nishimura. This is an open access article distributed under the Creative Commons Attribution License, which permits unrestricted use, distribution, and reproduction in any medium, provided the original work is properly cited.

Cerebral aneurysms (CAs) have a high prevalence and can cause a lethal subarachnoid hemorrhage. Currently, CAs can only be treated with invasive surgical procedures. To unravel the underlying mechanisms of CA formation and to develop new therapeutic drugs for CAs, animal models of CA have been established, modified, and analyzed. Experimental findings from these models have clarified some of the potential mechanisms of CA formation, especially the relationship between hemodynamic stress and chronic inflammation. Increased hemodynamic stress acting at the site of bifurcation of cerebral arteries triggers an inflammatory response mediated by various proinflammatory molecules in arterial walls, inducing pathological changes in the models similar to those observed in the walls of human CAs. Findings from animal studies have provided new insights into CA formation and may contribute to the development of new therapeutic drugs for CAs.

\section{Introduction}

Vascular diseases represent an important problem due to their potential severity and increasing prevalence. Many series of studies, especially those on atherosclerosis, have contributed to reduce the mortality rate of vascular diseases and to improve the patients' quality life [1]. Unfortunately, it is not a case in subarachnoid hemorrhage (SAH), which is a potentially lethal disease with a mortality rate of up to $50 \%$ and is responsible for more than 30,000 patient deaths per year in the United States $[2,3]$. Furthermore, SAH can cause a considerable cognitive impairment that results in restricted social activity, even in patients who recover well and successfully return to their daily lives [4]. Therefore, the prevention of SAH is a socially important issue. A cerebral aneurysm (CA) is the primary cause of spontaneous SAH and can be found in approximately $1 \%-5 \%$ of the general population $[3,5-7]$. Currently, surgical procedures such as microsurgical clipping and endovascular coiling are the only therapeutic modalities thought to prevent the rupture of preexisting CAs [6]. However, because of the inescapable risk of complications, surgical treatment is recommended only for a selected group of patients with an estimated high risk of rupture. This means that many patients with CAs are followed without any effective treatment, while managing potential risk factors for rupture $[5,8]$. Given the severity of SAH after the rupture of CAs, there is a strong demand for the development of a medical treatment for CA.

In the present paper, we review the current knowledge of the underlying mechanisms of CA formation, mainly derived from animal models and discuss the development, clinical relevance, usefulness, and future potential of animal models of CA.

\section{Findings and Limitations of Human Studies}

There is considerable interest in elucidating the underlying mechanisms of CA formation in order to develop less invasive medical treatments. For this purpose, many human studies, including the histopathological analysis of surgically dissected CAs or cerebral arteries, gene linkage analysis, and epidemiological analysis, have been performed and 
achieved a measure of success. These studies have revealed certain elements that may be important in the pathogenesis of CAs. Here, a CA is characterized as the blooming of cerebral arterial walls, especially at the bifurcation site, that is accompanied by the disruption of the internal elastic lamina and degenerative changes in arterial walls represented by the thinning of the media and the loss of medial smooth muscle cells $[9,10]$.

Aneurismal SAH has a familial clustering or a difference in race, suggesting the genetic influence. Certainly, gene linkage analyses have identified some of the susceptibility loci or genes that increase the risk for the development and rupture of CAs [11-24]. Most identified genes are pro-inflammatory genes or extracellular matrix-related genes, suggesting that an inflammatory process or the turnover of the extracellular matrix is actively involved in the pathogenesis of CAs.

Epidemiological analyses have identified some risk factors for the enlargement or rupture of CAs, most notably hypertension $[8,25,26]$, which increases the hemodynamic stress at the bifurcation site of cerebral arteries and potentially contributes to the formation of CAs through the induction of mechanical damage in the arterial wall [9]. These studies provided epidemiological support for a hypothesis derived from the computational simulation of blood flow at a prospective CA formation site which suggest that hemodynamic stress triggers CA formation [27-30].

Histopathological studies of human CA tissues have implicated the involvement of inflammatory processes in the pathogenesis of CA. The increased expressions of proinflammatory factors such as collagenolytic matrix metalloproteinases (MMPs) [31-33], vascular cell adhesion molecule-1 (VCAM-1) [34], tumor necrosis factor-alpha (TNF- $\alpha$ ) [35], monocyte chemoattractant protein-1 (MCP1) [36], and mitogen-activated protein kinases (MAPKs) [37, 38] have been found in human CA walls using immunostaining, Western blot, or PCR analysis. Further, histological studies also revealed the infiltration of macrophages and $\mathrm{T}$ cells [34], complement or immunoglobulin deposition and activation $[34,39]$, and apoptotic cell death in CA walls $[40,41]$, suggesting the active involvement of inflammation in CA formation.

As described above, studies using human samples have contributed to the understanding of the underlying mechanisms of CA formation; however, these studies also have several important limitations. First, microsurgical dissection of CA may increase the rupture. Second, CA formation is thought to be influenced by the genetic background as in the Japanese and Finish populations which are known to have a significantly higher incidence of CAs than other populations [4]. CAs can also exhibit familial clustering $[5,8,25]$. Taken together, the complexity of this problem may render the analysis of CA formation difficult. Third, we can only evaluate human CA samples at certain time points, and we can not assess sequential changes of CA formation. To overcome these limitations and to more systematically examine the underlying mechanisms of CA formation, animal models of CAs have been demanded and developed.

\section{Overview of Animal Models of CA}

Animal models can reduce the complexity of genetic analysis because of their homogeneous genetics and bring ease of manipulation through the availability of molecular biological tools. Currently, there are 2 major classes of animal models of CA. First are the animal models that are used to evaluate the underlying mechanisms of CA formation, which will be described in detail later on in this review. Second are the models mainly used to determine the technical proficiency of endovascular devices or to assess histopathological changes after endovascular treatment; however, this class is not used to determine the underlying mechanisms of CA formation. These models are surgically induced by creating a venous pouch mainly in the carotid artery of dogs or pigs [42-45].

\section{Animal Models of CA Induced by Increased Hemodynamics}

Several studies attempted to develop an animal model of $\mathrm{CA}$ in which the histopathological features of human CA were reproduced, for example, the disruption of the internal elastic lamina and the degeneration of the media, before Hashimoto et al. reported their first rat model of CA [46]. Previous attempts involved the use of various compounds including a nitrogen mustard and cyanoacrylate, in order to degenerate the arterial walls, for example, [47-49], but failed to induce CAs with similar pathological characteristics to human ones.

Hashimoto et al. successfully induced CAs at the bifurcation site of cerebral arteries of rats in 1978 by increasing hemodynamics on the basis of the hypothesis that CA was formed at fragile arterial walls loaded with a high hemodynamic stress [46]. They thoughtfully used the lathyrogen, $\beta$ aminopropionitrile (BAPN), which inhibits the cross-linking of collagen and elastin fibers $[50,51]$, to weaken arterial walls. The oral administration of BAPN to animals degenerates arterial walls and, as a result, occasionally induces an arterial dissection (angiolathyrism) [52, 53]. To further increase hemodynamic stress with systemic hypertension, they ligated the unilateral common carotid artery, performed a unilateral nephrectomy, administered deoxycorticosterone, and increased salt intake in BAPN-treated rats. CAs were thus spontaneously induced at the bifurcation sites of the cerebral arteries [46]. Histopathological studies of the induced CAs demonstrated that their pathological features were in accordance with those of human CAs [54]. This group also examined the effects of hemodynamic stress on CA formation by comparing the bilateral or unilateral ligation of carotid artery and confirmed that hemodynamic stress greatly influenced CA formation [29].

The CA rat model has since undergone several modifications. Currently, CAs are induced by the unilateral ligation of the common carotid artery with induced hypertension by the unilateral ligation of the renal artery and increased salt intake with or without BAPN treatment (Figure 1). BAPN treatment enhances the incidence of CA formation and the size of the induced CAs [46, 55]; however, it also disturbs normal collagen synthesis and the turnover 


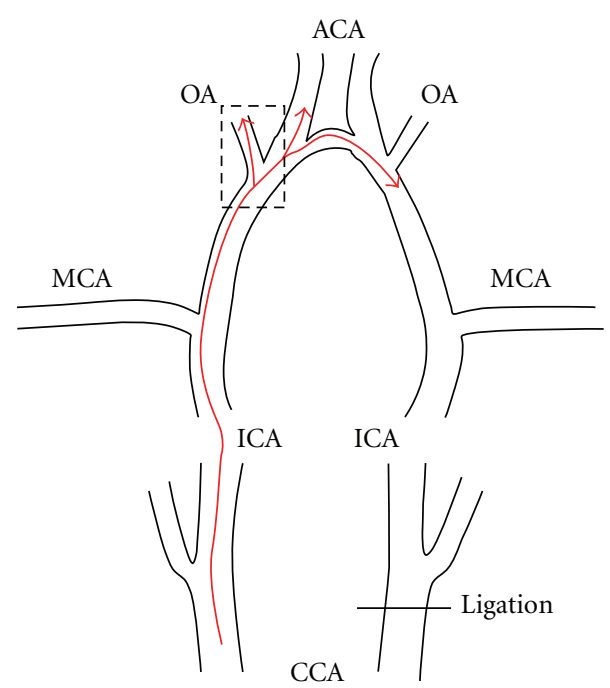

(a)

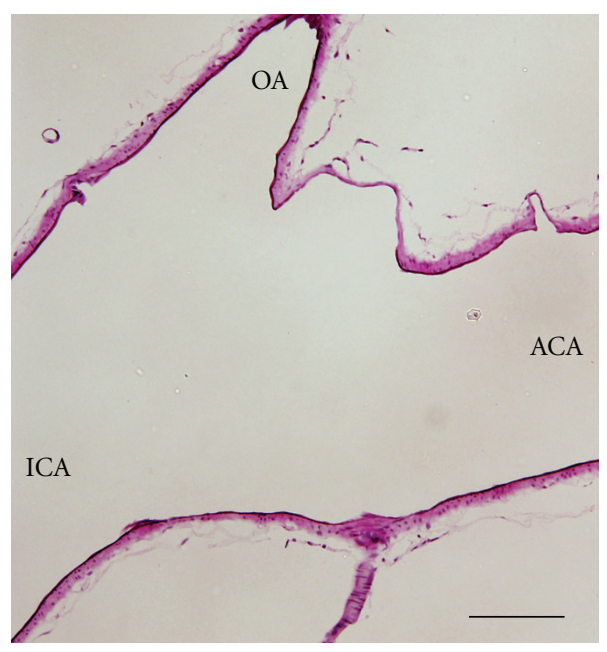

(c)

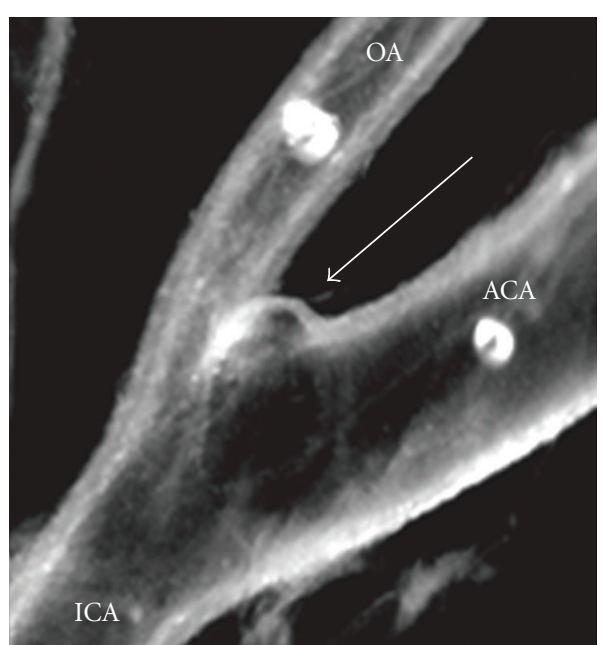

(b)

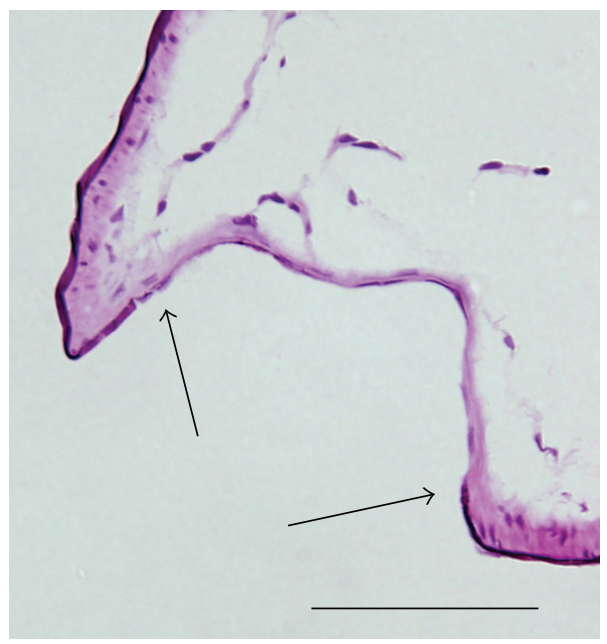

(d)

FIGURE 1: Cerebral aneurysm (CA) formation in the rat model. (a) The method for CA induction. As illustrated, the unilateral common carotid artery (CCA) is ligated to induce a compensatory increase in cerebral blood flow in the contralateral internal carotid artery (ICA). As a result of increased hemodynamics, a CA is induced at the anterior cerebral artery-(ACA-) olfactory artery (OA) bifurcation (indicated by the boxed region). MCA: middle cerebral artery. (b) Macroscopic image of the dissected ACA-OA bifurcation indicated as the boxed region in (a). Note that a CA is induced at this bifurcation (arrow). (c, d) Histopathological examination of induced CA by Elastica van Gieson staining. Higher magnified image of CA walls is shown in (d). Note that the disruption of the internal elastic lamina (arrow) and the degeneration of the media, which are histopathological features of human CA walls, are observed. Bar: $50 \mu \mathrm{m}$.

of the extracellular matrix. Further, Nagata et al. have also demonstrated that BAPN treatment and salt overloading have a synergistic effect on CA formation [56].

A mouse model of CA was successfully established in 2002 by the unilateral ligation of the carotid artery and induced hypertension [57]. The histopathological examination of murine CAs by electron microscopy shows the disrupted internal elastic lamina and degenerative changes in the media, as observed in human CAs [57]. As mice are more resistant to induced hypertension and vascular inflammation, more time is needed to induce CAs and their incidence is less than in the rat model [57, 58]. Technical advancements in molecular biology have enabled the generation of genetically modified mice. Increased availability of molecular biological tools in this species allows us to genetically analyze the underlying mechanisms of CA formation using various gene-deficient mice. These mice are particularly useful to analyze the contribution of particular genes to disease pathogenesis.

Hashimoto et al. have also successfully induced CAs in monkeys (Macaca fascicularis) by the ligation of the common carotid artery and systemic hypertension with BAPN treatment [59]. Histopathological studies revealed that the CAs induced in primates had similar characteristics as in human CAs [60]. The usage of the primate model is limited due to ethical concerns; however, since rats and mice 


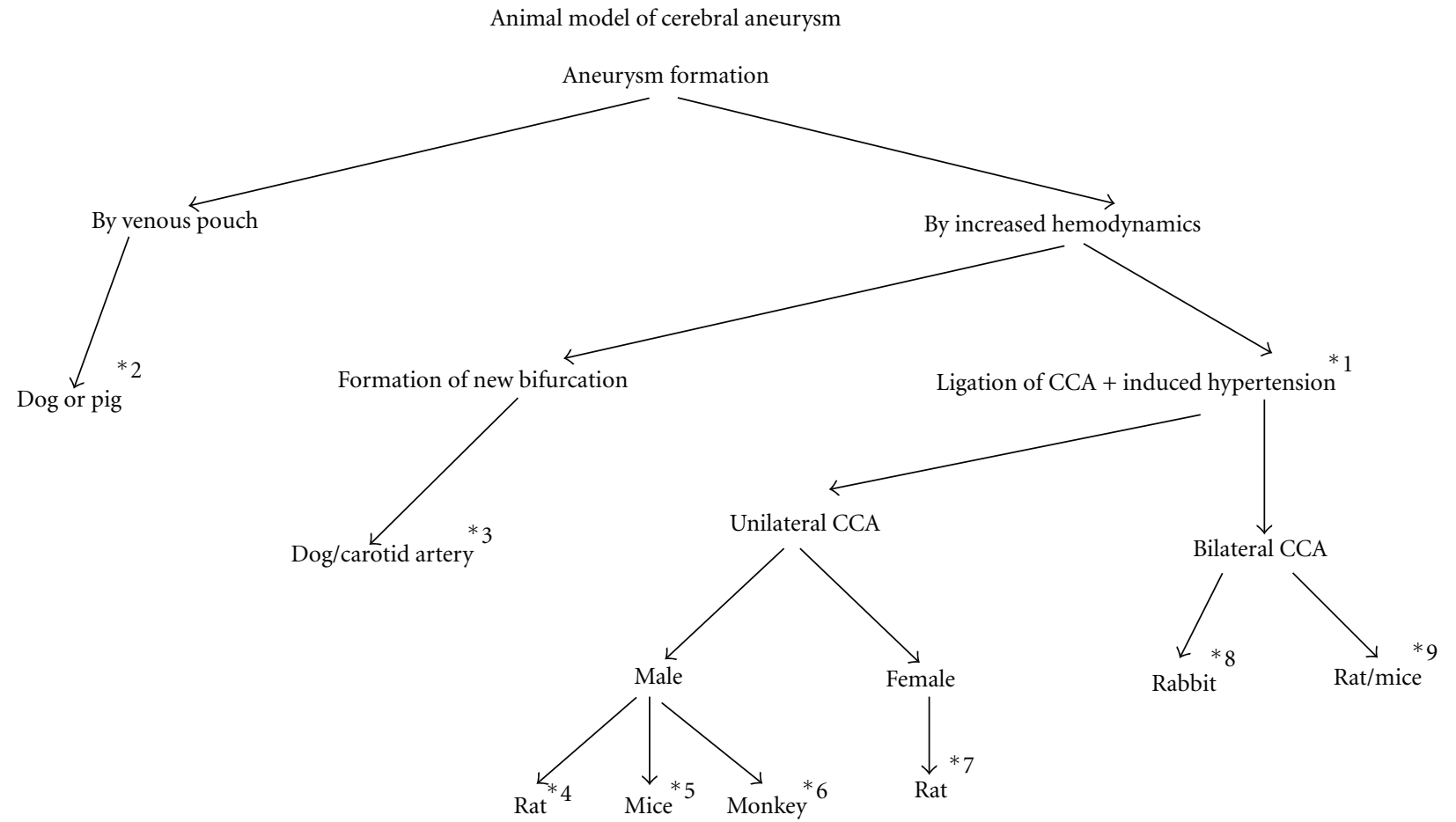

FIGURE 2: Brief summary of animal models of CA used in recent experimental studies. A brief summary of animal models used in recent experiments is shown. The particularities, advantages, and disadvantages of each model are as follows. * 1 : Induced hypertension can be achieved by several methods such as the ligation of the renal artery, high salt diet intake, deoxycorticosterone treatment, and angiotensin II injection. *2: This model is mainly used for the development of endovascular devices and not for the analysis of the molecular mechanisms of CA formation. *3: The new bifurcation is created at the carotid artery by side-to-end ligation. This model is suitable for the analysis of hemodynamics-induced CAs, but the hemodynamic stress is greater than in the intracranial arteries where the CAs are formed. ${ }^{*} 4$ : CAs can be induced with a high frequency and with a low risk, but they seldom rupture. BAPN treatment enlarges the size of the induced CAs. ${ }^{*} 5$ : BAPN intake or local elastase injection is essential for the induction of CAs. Lipopolysaccharide can also enhance CA formation and huge CAs can sometimes be observed. *6: It takes over 1 year to induce CAs, but their incidence is high. ${ }^{*} 7$ : It is necessary to perform bilateral oophorectomy to effectively induce CAs. ${ }^{*} 8$ : Large CAs are frequently induced in the posterior circulation. This model has the potential to be used in studies linking hemodynamics and molecular expression. *9: Bilateral CCA ligation results in high mortality, but can induce large CAs in the posterior circulation. CCA: common carotid artery.

are more resistant to arterial inflammation than primates and monkeys and humans are closely related species, a monkey model may still be necessary in some situations.

Several other groups have also applied the induced hypertension/lathyrism model [30, 52, 61-66]. We have mainly used male rats or mice as CA models to exclude the influence of sex hormones. Jamous et al. recently induced CAs in female rats through carotid ligation, induced hypertension, and bilateral oophorectomy [63]. The sex hormone estrogen has a protective effect on endothelial cell function and may therefore influence CA formation [67]. Indeed, they demonstrated that bilateral oophorectomy increased the size of the induced CAs by decreasing the levels of estrogen [63]. Furthermore, they examined the protective effect of estrogen on CA formation by applying hormone replacement therapy to their rat model and confirmed that hormone replacement therapy could attenuate the increased formation of CAs by bilateral oophorectomy [62].

Gao et al. induced CAs in New Zealand white rabbits by increasing hemodynamic stress through the bilateral ligation of the carotid arteries [61]. He and his colleagues selected rabbits because the arterial diameter of rats and mice is too small to reproducibly perform a hydrokinetic analysis of hemodynamics during CA formation. They demonstrated that increased hemodynamic stress triggered CA formation and that the induction of CAs was well correlated with the increase of blood flow in the basilar artery.

Meng et al., who was the corresponding author of Gao's paper, further reported the correlation of hemodynamic stress with CA formation using a canine model [30]. They induced CAs by surgically creating a new bifurcation site using the carotid artery of dogs and measured the hemodynamic stress in situ. As a result, the high wall shear stress and high wall shear stress gradient induced pathological changes resembling human $\mathrm{CA}$, for example, the disruption of the internal elastic lamina and the loss of medial smooth muscle cells [30]. The canine models were used to unravel the correlation of hemodynamic stress with CA formation using a different concept than our models, which are designed to study the molecular basis of CA formation.

We and other researchers have modified the present CA models and tried to develop novel models that are designed 


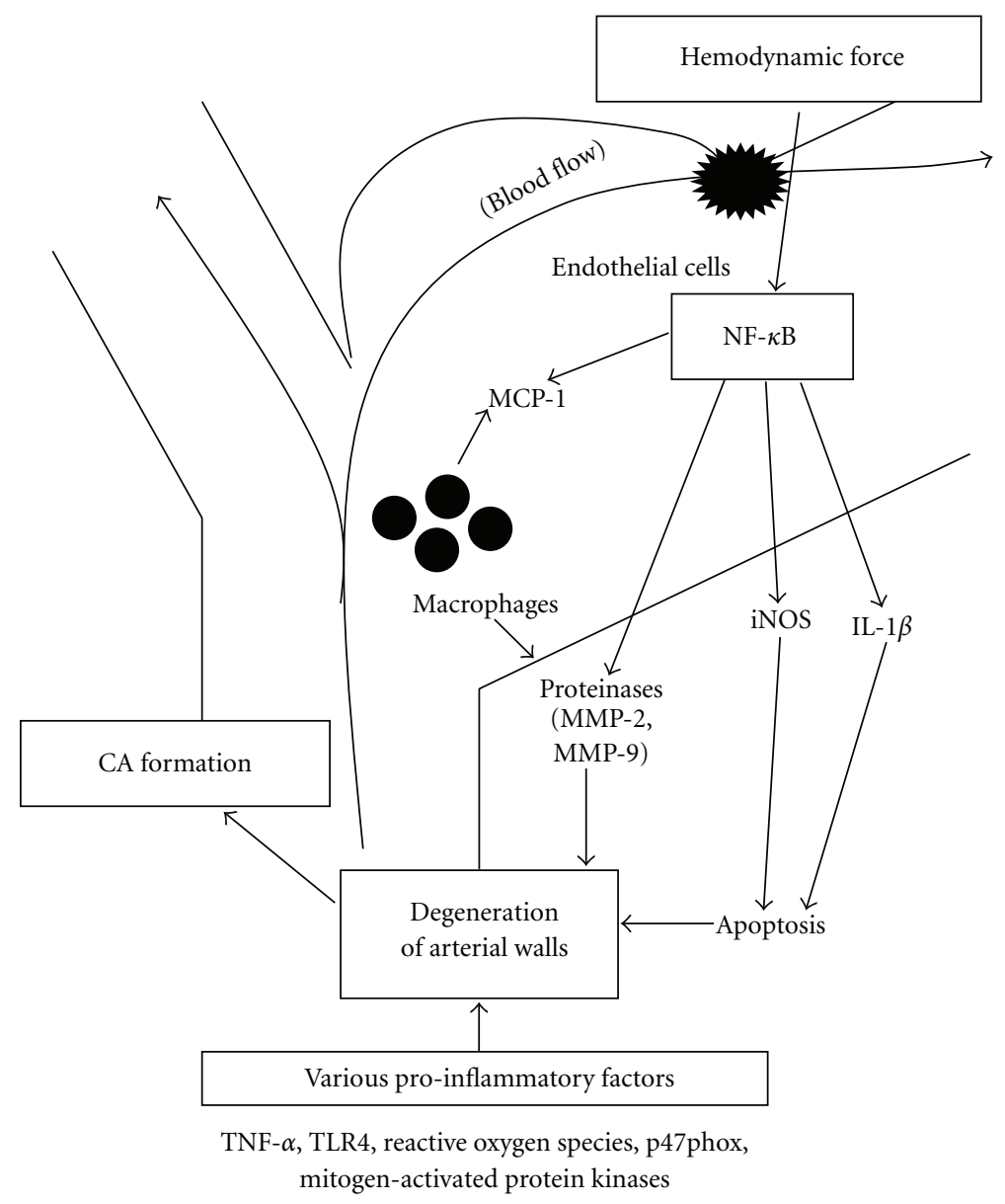

FIGURE 3: Proposed mechanisms of CA formation from human and animal studies. Proposed mechanisms of CA formation from human and animal studies are summarized and illustrated.

to answer a question (Figure 2). In the near future, we hope that more desirable models of CAs will be developed to reveal the mechanisms of CA formation in more detail.

\section{Exploratory Pathophysiology of CAs from Animal Studies}

Animal models of CA have provided us with insights into the underlying mechanisms of CA formation, and we can propose a hypothesis for the molecular basis of CA formation from the experimental findings of animal models (Figure 3 ). Here, we will summarize the experimental findings of CA formation.

On the basis of the finding that inflammatory cells, especially macrophages, are observed in CA walls in humans [34], the infiltration of inflammatory cells in CA walls was assessed using a rat model. The primary inflammatory cells in rat CA walls are macrophages, in good agreement with observations in human CA walls $[68,69]$, and their number gradually increases during CA formation, suggesting that macrophage-mediated inflammation may contribute to CA formation [68]. Macrophages secrete various tissuedestructive proteinases and cytokines, which can degenerate the components of the extracellular matrix of arterial walls, especially collagen and elastin, and are hypothesized to contribute to CA formation, especially the enlargement of CAs through the weakening of CA walls. Indeed, macrophages secrete collagenolytic proteinases, MMP-2 and MMP-9 in CA walls of rats $[68,70]$, and enhanced collagenolytic activity is detected in CA walls of rat models by in situ zymography [68, 69] or gelatin zymography [71-74]. Consistent with these observations, the oral administration of MMP inhibitors to rat models or a deficiency of MMP-9 results in the decreased formation of CAs $[68,69]$. Another group of proteinases, cysteine cathepsins, are also upregulated in rat CA walls and their activities are significantly elevated in comparison with those in control arterial walls [75]. A specific inhibitor of cathepsins effectively suppresses CA formation [75]. These results suggest that proteinases actively participate in the pathogenesis of CA and promote CA formation.

Macrophages are recruited to inflammatory sites by chemoattractants, for example, MCP-1 [76, 77]. In CA walls of rat models, the expression of MCP-1 is upregulated in intimal endothelial cells at the early stage of CA formation [58] and in whole arterial walls, including infiltrating inflammatory cells, at the late stage $[58,70]$. These findings are in accord with previous human studies in which the expression of MCP-1 mRNA was confirmed in human 
CA specimens by in situ hybridization [36]. Further, in MCP-1-deficient mice, in which macrophage infiltration of CA walls is abolished, CA formation is inhibited with the suppression of inflammation [58]. The upregulation of various pro-inflammatory molecules in human CA walls has been reported and gene microarray data also support these observations [17, 78].

We identified the upregulation of pro-inflammatory genes during CA formation in CA walls of rat models using microarray analysis and adaptor-tagged competitivePCR analysis [79, 80]. Most of the upregulated genes can be categorized as proteinases, cytokines, adhesion molecules, reactive oxygen species, members of the apoptotic cascade, and complements, suggesting that the inflammatory response in arterial walls plays a crucial role in CA formation [79].

The genetic contribution to CA formation has also been examined using animal models. In rats, the upregulation of reactive oxygen species-producing enzymes such as p47phox [81], superoxide-related genes such as NOX4 or Rac1 [70], cytokines such as interleukin- $1 \beta$ (IL-1 $\beta$ ) [82], and inducible nitric oxide synthase (iNOS) [83] is confirmed in CA walls. However, contradictory results for the contribution of the renin-angiotensin system (RAS) in CA walls have been reported. Tada et al. reported in the induced CA walls of female rat models that the expression of angiotensin converting enzyme (ACE) was upregulated as determined by RT-PCR analysis, and the final product of RAS, angiotensin II production, was also increased in CA walls as determined by immunostaining [70]. On the contrary, our group found that angiotensin II receptor type 1 (AT1R) was not induced in CA walls of male rats by immunostaining and, consistently, the administration of an AT1R blocker (ARB) failed to inhibit CA formation [84]. In human CA walls, Ohkuma et al. reported the decreased expression of ACE and AT1R by RT-PCR analysis and immunostaining [85]. It is not clear why these differences occur and future studies should address this issue.

In mice, the role of a particular gene on CA formation can be assessed more clearly by applying gene-deficient mice to the models. Mice deficient in MMP-2 or -9 [69], tissue inhibitor of MMP (TIMP)-1 or -2 [73], MCP-1 [58], p47phox [55], toll-like receptor-4 (TLR-4) [86], NF- $\kappa$ B p50 subunit [87], IL-1 $\beta$ [82], apolipoprotein E (ApoE) [88], or iNOS [89] have been applied to CA models revealing the involvement of each gene in the pathogenesis of CAs. In summary, MMP-9, MCP-1, p47phox, TLR-4, NF- $\kappa$ B p65 subunit, IL- $1 \beta$, and iNOS promote CA formation, while TIMP- 1 and TIMP-2 suppresses CA formation.

These experimental results suggest that chronic inflammation in arterial walls plays the important role in CA formation. We have proposed that the transcription factor $\mathrm{NF}-\kappa \mathrm{B}$ is a critical mediator due to its transcriptional regulation of various pro-inflammatory genes in CA walls including MMPs, iNOS, IL- $1 \beta$, and MCP-1, which have been shown to be involved in the pathogenesis of CA $[58,68,82$, $83,87,89]$. NF- $\kappa \mathrm{B}$ is phosphorylated and activated first in the endothelial cells of CA walls at the early stage of CA formation and then throughout CA walls at the late stage
[88]. We have hypothesized that NF- $\kappa \mathrm{B}$ activation in the endothelial cell layer is due to hemodynamic stress, which is thought to be a trigger for CA formation $[27,29,90-$ 94]. This hypothesis is in line with the experimental findings that NF- $\kappa \mathrm{B}$ is activated in endothelial cells by hemodynamic stress in vitro [95] and that high hemodynamic stress induces the expression of MMP-2, -9, iNOS, and IL- $1 \beta$ in CA walls created in the carotid arteries of a canine model [96]. The inhibition of NF- $\kappa$ B transcriptional activity by decoy oligodeoxynucleotides [97] or a deficiency in the p50 subunit of NF- $\kappa$ B results in the remarkable inhibition of CA formation by the suppression of pro-inflammatory gene expression and macrophage infiltration in CA walls, suggesting the critical role of $\mathrm{NF}-\kappa \mathrm{B}$ activation in CA formation [88].

\section{Limitations of the Existing CA Models and the Necessity for Further Models}

As described in the previous sections, animal models of CA have been used to demonstrate some of the important mechanisms of CA formation and identified therapeutic targets for the treatment of human CAs (Figure 3); however, there is a fundamental problem to be addressed in animal models. It is beyond question that the rupture of CAs and the subsequent $\mathrm{SAH}$ is a devastating event and should be prevented; therefore, the prevention of CA rupture is a critical target. For this reason, animal models of CAs are needed where CAs have a high likelihood of spontaneous rupture. Unfortunately, there are no established models with this feature. In our rat models, the rupture rate is only $3 \%$ during 3 months after CA induction. We have preliminarily examined several compounds and modified the procedures to induce CAs; however, we failed to establish a rat model with a high incidence of rupture and have never observed the spontaneous rupture of CAs in mice. Recently, Nuki et al. reported that they stereotaxically injected an elastase into the basal cistern in combination with the continuous infusion of angiotensin II to induce CAs in mice [69]. In their report, these induced CAs in murine models spontaneously ruptured, although the exact incidence of rupture was not made clear [69]. Therefore, it is essential that an animal model is developed in which CAs spontaneously and frequently rupture.

\section{Future Perspectives: From the Results of Animal Studies to Human Treatment}

Extensive studies using animal models of CAs are, of course, designed to unravel the underlying mechanisms of CA formation and to establish new therapeutic drugs for unruptured CAs in humans. As described in the previous sections, we have identified NF- $\kappa \mathrm{B}$ as one of the factors closely related to CA formation and a potential therapeutic target $[87,98,99]$, therefore, we have examined the preventive effects of several drugs with anti-NF- $\kappa \mathrm{B}$ activity on the enlargement of CAs induced in a rat model [71, $72,74,81,84]$. Among several candidate drugs, the class of statins, a powerful cholesterol-lowering drug, have been 
found to effectively prevent the enlargement of CAs induced in rats $[71,74]$. The oral administration of statin to rats significantly suppresses the expression of pro-inflammatory genes in CA walls [74] through its anti-NF- $\kappa$ B effect well known as a pleiotropic effect [100]. As a result, the chronic inflammation in CA walls, transcriptionally regulated by NF$\kappa \mathrm{B}$, is inhibited. These results, combined with experimental findings indicating that chronic inflammation contributes to $\mathrm{CA}$ formation, suggest that statin can suppress the enlargement of CAs. In present models, we can not assess the rupture of CAs because of their low incidence of spontaneous rupture; however, statin effectively prevents the degenerative changes of CA walls, suggesting a preventive effect of statin on the rupture of CAs. Furthermore, we and others have independently demonstrated that 3 different kinds of statins prevent the formation and enlargement of CAs induced in rat models, indicating that the preventive effect of statins on the enlargement of CAs is due to their class effect $[71,74,101]$. These results suggest the therapeutic potential of statins as drugs for CAs in humans. Based on these experimental findings, a case-control study to examine the preventive effect of statins on the rupture of human CAs is currently in progress. Other candidates as therapeutic drugs for CA treatment, for example, Ibudilast or a phosphodiesterase4 inhibitor, have been examined using rat CA models with focus on their anti-inflammatory effects $[66,70,102]$.

\section{Conclusions}

SAH caused by the rupture of a pre-existing CA is a potentially devastating and socially restricting event. To unravel the underlying mechanisms of CA formation and to develop a new class of therapeutic drugs to prevent rupture, animal models of CAs have been established. Experimental results from animal models and findings from human specimens have revealed some of the important mechanisms underlying CA formation. Increased hemodynamic stress at the bifurcation site of the cerebral arteries is considered to play a crucial role in CA formation by inducing inflammatory responses in arterial walls. Inflammation in cerebral arterial walls triggered by hemodynamic stress is considered to disrupt the internal elastic lamina and degenerate the media. As a result of the increased hemodynamic stress and inflammatory responses in cerebral arteries, CA is induced. Through recent experimental findings, we have identified NF- $\kappa \mathrm{B}$ as a potential therapeutic target for CA. The inhibition of NF- $\kappa$ B activity in animal models, significantly suppress CA formation, suggesting that anti-NF- $\kappa \mathrm{B}$ drugs can potentially be therapeutic drugs for CAs. In the future, there is a hope that, through increasing use and extensive studies by animal models of CAs, the elucidation of the underlying mechanisms of CA formation allows the pharmacological prevention of SAH.

\section{Acknowledgment}

The authors extend our sincere gratitude to our collaborators for their kind advice and efforts to develop and modify CA models.

\section{References}

[1] R. Ross, "Atherosclerosis—an inflammatory disease," New England Journal of Medicine, vol. 340, no. 2, pp. 115-126, 1999.

[2] V. L. Feigin and M. Findlay, "Advances in subarachnoid hemorrhage," Stroke, vol. 37, no. 2, pp. 305-308, 2006.

[3] J. van Gijn, R. S. Kerr, and G. J. Rinkel, "Subarachnoid haemorrhage," Lancet, vol. 369, no. 9558, pp. 306-318, 2007.

[4] M. J. H. Wermer, H. Kool, K. W. Albrecht, and G. J. E. Rinkel, "Subarachnoid hemorrhage treated with clipping: long-term effects on employment, relationships, personality, and mood," Neurosurgery, vol. 60, no. 1, pp. 91-97, 2007.

[5] P. M. White and J. M. Wardlaw, "Unruptured intracranial aneurysms: detection and management," Journal of Neuroradiology, vol. 30, no. 5, pp. 336-350, 2003.

[6] D. O. Wiebers, J. P. Whisnant, J. Huston III et al., "Unruptured intracranial aneurysms: natural history, clinical outcome, and risks of surgical and endovascular treatment," Lancet, vol. 362, no. 9378, pp. 103-110, 2003.

[7] J. Van Gijn and G. J. E. Rinkel, "Subarachnoid haemorrhage: diagnosis, causes and management," Brain, vol. 124, no. 2, pp. 249-278, 2001.

[8] M. Clarke, "Systematic review of reviews of risk factors for intracranial aneurysms," Neuroradiology, vol. 50, no. 8, pp. 653-664, 2008.

[9] S. Inci and R. F. Spetzler, "Intracranial aneurysms and arterial hypertension: a review and hypothesis," Surgical Neurology, vol. 53, no. 6, pp. 530-542, 2000.

[10] W. I. Schievink, "Intracranial aneurysms," New England Journal of Medicine, vol. 336, no. 1, pp. 28-40, 1997.

[11] E. Biros and J. Golledge, "Meta-analysis of whole-genome linkage scans for intracranial aneurysm," Neuroscience Letters, vol. 431, no. 1, pp. 31-35, 2008.

[12] T. Foroud, L. Sauerbeck, R. Brown et al., "Genome screen to detect linkage to intracranial aneurysm susceptibility genes: the familial intracranial aneurysm (FIA) study," Stroke, vol. 39, no. 5, pp. 1434-1440, 2008.

[13] A. Helgadottir, G. Thorleifsson, K. P. Magnusson et al., "The same sequence variant on $9 \mathrm{p} 21$ associates with myocardial infarction, abdominal aortic aneurysm and intracranial aneurysm," Nature Genetics, vol. 40, no. 2, pp. 217-224, 2008.

[14] K. Inoue, Y. Mineharu, S. Inoue et al., "Search on chromosome 17 centromere reveals TNFRSF13B as a susceptibility gene for intracranial aneurysm: a preliminary study," Circulation, vol. 113, no. 16, pp. 2002-2010, 2006.

[15] D. Krex, S. Fortun, E. Kuhlisch, H. K. Schackert, and G. Schackert, "The role of endothelial nitric oxide synthase (eNOS) genetic variants in European patients with intracranial aneurysms," Journal of Cerebral Blood Flow and Metabolism, vol. 26, no. 10, pp. 1250-1255, 2006.

[16] D. Krex, H. Röhl, I. R. König, A. Ziegler, H. K. Schackert, and G. Schackert, "Tissue inhibitor of metalloproteinases$1,-2$, and-3 polymorphisms in a white population with intracranial aneurysms," Stroke, vol. 34, no. 12, pp. 28172821, 2003.

[17] B. Krischek, H. Kasuya, A. Tajima et al., "Network-based gene expression analysis of intracranial aneurysm tissue reveals role of antigen presenting cells," Neuroscience, vol. 154, no. 4, pp. 1398-1407, 2008.

[18] Y. Mineharu, K. Inoue, S. Inoue et al., "Association analyses confirming a susceptibility locus for intracranial aneurysm at chromosome 14q23," Journal of Human Genetics, vol. 53, no. 4, pp. 325-332, 2008. 
[19] Y. Mineharu, K. Inoue, S. Inoue et al., "Model-based linkage analyses confirm chromosome $19 \mathrm{q} 13.3$ as a susceptibility locus for intracranial aneurysm," Stroke, vol. 38, no. 4, pp. 1174-1178, 2007.

[20] J. M. Olson, S. Vongpunsawad, H. Kuivaniemi et al., "Search for intracranial aneurysm susceptibility gene(s) using Finnish families," BMC Medical Genetics, vol. 3, article 7, 2002.

[21] A. K. Ozturk, B. V. Nahed, M. Bydon et al., "Molecular genetic analysis of two large kindreds with intracranial aneurysms demonstrates linkage to 11q24-25 and 14q23-31," Stroke, vol. 37, no. 4, pp. 1021-1027, 2006.

[22] Y. M. Ruigrok and G. J. E. Rinkel, "Genetics of intracranial aneurysms," Stroke, vol. 39, no. 3, pp. 1049-1055, 2008.

[23] D. Woo and J. Broderick, "Genetics of intracranial aneurysm," Journal of Stroke and Cerebrovascular Diseases, vol. 11, no. 5, pp. 230-240, 2002.

[24] T. Yoneyama, H. Kasuya, H. Onda et al., "Association of positional and functional candidate genes FGF1, FBN2, and LOX on 5q31 with intracranial aneurysm," Journal of Human Genetics, vol. 48, no. 6, pp. 309-314, 2003.

[25] V. L. Feigin, G. J. E. Rinkel, C. M. M. Lawes et al., "Risk factors for subarachnoid hemorrhage: an updated systematic review of epidemiological studies," Stroke, vol. 36, no. 12, pp. 27732780, 2005.

[26] S. Juvela, M. Porras, and K. Poussa, "Natural history of unruptured intracranial aneurysms: probability of and risk factors for aneurysm rupture," Journal of Neurosurgery, vol. 108, no. 5, pp. 1052-1060, 2008.

[27] M. S. Alnæs, J. Isaksen, K. A. Mardal, B. Romner, M. K. Morgan, and T. Ingebrigtsen, "Computation of hemodynamics in the circle of Willis," Stroke, vol. 38, no. 9, pp. 2500-2505, 2007.

[28] M. A. Castro, C. M. Putman, M. J. Sheridan, and J. R. Cebral, "Hemodynamic patterns of anterior communicating artery aneurysms: a possible association with rupture," American Journal of Neuroradiology, vol. 30, no. 2, pp. 297-302, 2009.

[29] N. Hashimoto, H. Handa, I. Nagata, and F. Hazama, "Experimentally induced cerebral aneurysms in rats: V. Relation of hemodynamics in the circle of Willis to formation of aneurysms," Surgical Neurology, vol. 13, no. 1, pp. 41-45, 1980.

[30] H. Meng, Z. Wang, Y. Hoi et al., "Complex hemodynamics at the apex of an arterial bifurcation induces vascular remodeling resembling cerebral aneurysm initiation," Stroke, vol. 38, no. 6, pp. 1924-1931, 2007.

[31] G. Bruno, R. Todor, I. Lewis, and D. Chyatte, "Vascular extracellular matrix remodeling in cerebral aneurysms," Journal of Neurosurgery, vol. 89, no. 3, pp. 431-440, 1998.

[32] D. Jin, J. Sheng, X. Yang, and B. Gao, "Matrix metalloproteinases and tissue inhibitors of metalloproteinases expression in human cerebral ruptured and unruptured aneurysm," Surgical Neurology, vol. 68, no. 6, supplement 2, pp. S11-S16, 2007.

[33] S. C. Kim, M. Singh, J. Huang et al., "Matrix metalloproteinase-9 in cerebral aneurysms," Neurosurgery, vol. 41, no. 3, pp. 642-647, 1997.

[34] D. Chyatte, G. Bruno, S. Desai, and D. R. Todor, "Inflammation and intracranial aneurysms," Neurosurgery, vol. 45, no. 5, pp. 1137-1147, 1999.

[35] T. Jayaraman, V. Berenstein, X. Li et al., "Tumor necrosis factor $\alpha$ is a key modulator of inflammation in cerebral aneurysms," Neurosurgery, vol. 57, no. 3, pp. 558-563, 2005.
[36] Y. Cao, J. Zhao, S. Wang, H. Zhong, and B. Wu, "Monocyte chemoattractant protein-1 mRNA in human intracranial aneurysm walls," Zhonghua Yu Fang Yi. Xue. Za Zhi, vol. 36, no. 7, pp. 519-521, 2002.

[37] E. Laaksamo, R. Tulamo, M. Baumann et al., "Involvement of mitogen-activated protein kinase signaling in growth and rupture of human intracranial aneurysms," Stroke, vol. 39, no. 3, pp. 886-892, 2008.

[38] Y. Takagi, M. Ishikawa, K. Nozaki et al., "Increased expression of phosphorylated c-Jun amino-terminal kinase and phosphorylated c-Jun in human cerebral aneurysms: role of the c-Jun amino-terminal kinase/c-Jun pathway in apoptosis of vascular walls," Neurosurgery, vol. 51, no. 4, pp. 997-1004, 2002.

[39] R. Tulamo, J. Frosen, S. Junnikkala et al., "Complement activation associates with saccular cerebral artery aneurysm wall degeneration and rupture," Neurosurgery, vol. 59, no. 5, pp. 1069-1076, 2006.

[40] F. Guo, Z. Li, L. Song et al., "Increased apoptosis and cysteinyl aspartate specific protease-3 gene expression in human intracranial aneurysm," Journal of Clinical Neuroscience, vol. 14, no. 6, pp. 550-555, 2007.

[41] L. Pentimalli, A. Modesti, A. Vignati et al., "Role of apoptosis in intracranial aneurysm rupture," Journal of Neurosurgery, vol. 101, no. 6, pp. 1018-1025, 2004.

[42] A. Berenstein, J. K. Song, T. Tsumoto, K. Namba, and Y. Niimi, "Treatment of experimental aneurysms with an embolic-containing device and liquid embolic agent: feasibility and angiographic and histological results," Neurosurgery, vol. 64, no. 2, pp. 367-373, 2009.

[43] J. Raymond, T. Darsaut, I. Salazkin, G. Gevry, and F. Bouzeghrane, "Mechanisms of occlusion and recanalization in canine carotid bifurcation aneurysms embolized with platinum coils: an alternative concept," American Journal of Neuroradiology, vol. 29, no. 4, pp. 745-752, 2008.

[44] J. Raymond, D. Venne, S. Allas et al., "Healing mechanisms in experimental aneurysms: I. Vascular smooth muscle cells and neointima formation," Journal of Neuroradiology, vol. 26, no. 1, pp. 7-20, 1999.

[45] I. Yuki, D. Lee, Y. Murayama et al., "Thrombus organization and healing in an experimental aneurysm model. Part II. The effect of various types of bioactive bioabsorbable polymeric coils," Journal of Neurosurgery, vol. 107, no. 1, pp. 109-120, 2007.

[46] N. Hashimoto, H. Handa, and F. Hazama, "Experimentally induced cerebral aneurysms in rats," Surgical Neurology, vol. 10, no. 1, pp. 3-8, 1978.

[47] W. S. McCune, A. Samadi, and B. Blades, "Experimental aneurysms," Annals of Surgery, vol. 138, no. 2, pp. 216-218, 1953.

[48] S. Tominaga, "An experimental study of hemodynamic factors in the rupture of intracranial aneurysms," Archiv fur Japanische Chirurgie, vol. 35, no. 1, pp. 67-88, 1966.

[49] H. Troupp and T. Rinne, "Methyl-2-cyanoactylate (Eastman 910) in experimental vascular surgery with a note on experimental arterial aneurysms," Journal of Neurosurgery, vol. 21, pp. 1067-1069, 1964.

[50] A. Sampath Narayanan, R. C. Siegel, and G. R. Martin, "On the inhibition of lysyl oxidase by $\beta$-aminopropionitrile," Biochemical and Biophysical Research Communications, vol. 46, no. 2, pp. 745-751, 1972.

[51] S. S. Tang, P. C. Trackman, and H. M. Kagan, "Reaction of aortic lysyl oxidase with $\beta$-aminopropionitrile," Journal of Biological Chemistry, vol. 258, no. 7, pp. 4331-4338, 1983. 
[52] M. V. Barrow, C. F. Simpson, and E. J. Miller, "Lathyrism: a review," Quarterly Review of Biology, vol. 49, no. 2, pp. 101$128,1974$.

[53] H. Selye, "Lathyrism," Revue Canadienne de Biologie, vol. 16, no. 1, pp. 1-82, 1957.

[54] N. Hashimoto, H. Handa, and F. Hazama, "Experimentally induced cerebral aneurysms in rats: part III. Pathology," Surgical Neurology, vol. 11, no. 4, pp. 299-304, 1979.

[55] T. Aoki, H. Kataoka, R. Ishibashi, K. Nozaki, R. Morishita, and N. Hashimoto, "Reduced collagen biosynthesis is the hallmark of cerebral aneurysm: contribution of interleukin$1 \beta$ and nuclear factor- $\kappa \mathrm{B}$," Arteriosclerosis, Thrombosis, and Vascular Biology, vol. 29, no. 7, pp. 1080-1086, 2009.

[56] I. Nagata, H. Handa, and N. Hashimoto, "Experimentally induced cerebral aneurysms in rats: part IV. Cerebral angiography," Surgical Neurology, vol. 12, no. 5, pp. 419-424, 1979.

[57] M. Morimoto, S. Miyamoto, A. Mizoguchi, N. Kume, T. Kita, and N. Hashimoto, "Mouse model of cerebral aneurysm: experimental induction by renal hypertension and local hemodynamic changes," Stroke, vol. 33, no. 7, pp. 1911-1915, 2002.

[58] T. Aoki, H. Kataoka, R. Ishibashi, K. Nozaki, K. Egashira, and N. Hashimoto, "Impact of monocyte chemoattractant protein-1 deficiency on cerebral aneurysm formation," Stroke, vol. 40, no. 3, pp. 942-951, 2009.

[59] N. Hashimoto, C. Kim, H. Kikuchi, M. Kojima, Y. Kang, and F. Hazama, "Experimental induction of cerebral aneurysms in monkeys," Journal of Neurosurgery, vol. 67, no. 6, pp. 903905, 1987.

[60] C. Kim, H. Kikuchi, N. Hashimoto, and F. Hazama, "Histopathological study of induced cerebral aneurysms in primates," Surgical Neurology, vol. 32, no. 1, pp. 45-50, 1989.

[61] L. Gao, Y. Hoi, D. D. Swartz, J. Kolega, A. Siddiqui, and H. Meng, "Nascent aneurysm formation at the basilar terminus induced by hemodynamics," Stroke, vol. 39, no. 7, pp. 20852090, 2008.

[62] M. A. Jamous, S. Nagahiro, K. T. Kitazato, T. Tamura, K. Kuwayama, and K. Satoh, "Role of estrogen deficiency in the formation and progression of cerebral aneurysms. Part II: experimental study of the effects of hormone replacement therapy in rats," Journal of Neurosurgery, vol. 103, no. 6, pp. 1052-1057, 2005.

[63] M. A. Jamous, S. Nagahiro, K. T. Kitazato, J. Satomi, and K. Satoh, "Role of estrogen deficiency in the formation and progression of cerebral aneurysms. Part I: experimental study of the effect of oophorectomy in rats," Journal of Neurosurgery, vol. 103, no. 6, pp. 1046-1051, 2005.

[64] M. A. Jamous, S. Nagahiro, K. T. Kitazato et al., "Endothelial injury and inflammatory response induced by hemodynamic changes preceding intracranial aneurysm formation: experimental study in rats," Journal of Neurosurgery, vol. 107, no. 2, pp. 405-411, 2007.

[65] H. Meng, Y. Feng, S. H. Woodward et al., "Mathematical model of the rupture mechanism of intracranial saccular aneurysms through daughter aneurysm formation and growth," Neurological Research, vol. 27, no. 5, pp. 459-465, 2005.

[66] K. Yagi, Y. Tada, K. T. Kitazato, T. Tamura, J. Satomi, and S. Nagahiro, "Ibudilast inhibits cerebral aneurysms by downregulating inflammation-related molecules in the vascular wall of rats," Neurosurgery, vol. 66, no. 3, pp. 551-559, 2010 .
[67] H. J. Teede, "Sex hormones and the cardiovascular system: effects on arterial function in women," Clinical and Experimental Pharmacology and Physiology, vol. 34, no. 7, pp. 672676, 2007.

[68] T. Aoki, H. Kataoka, M. Morimoto, K. Nozaki, and N. Hashimoto, "Macrophage-derived matrix metalloproteinase-2 and -9 promote the progression of cerebral aneurysms in rats," Stroke, vol. 38, no. 1, pp. 162-169, 2007.

[69] Y. Nuki, T. L. Tsou, C. Kurihara, M. Kanematsu, Y. Kanematsu, and T. Hashimoto, "Elastase-induced intracranial aneurysms in hypertensive mice," Hypertension, vol. 54, no. 6, pp. 1337-1344, 2009.

[70] Y. Tada, K. T. Kitazato, T. Tamura et al., "Role of mineralocorticoid receptor on experimental cerebral aneurysms in rats," Hypertension, vol. 54, no. 3, pp. 552-557, 2009.

[71] T. Aoki, H. Kataoka, R. Ishibashi, K. Nozaki, and N. Hashimoto, "Simvastatin suppresses the progression of experimentally induced cerebral aneurysms in rats," Stroke, vol. 39, no. 4, pp. 1276-1285, 2008.

[72] T. Aoki, H. Kataoka, R. Ishibashi, K. Nozaki, and N. Hashimoto, "Nifedipine inhibits the progression of an experimentally induced cerebral aneurysm in rats with associated down-regulation of NF-kappa B transcriptional activity," Current Neurovascular Research, vol. 5, no. 1, pp. 37-45, 2008.

[73] T. Aoki, H. Kataoka, T. Moriwaki, K. Nozaki, and N. Hashimoto, "Role of TIMP-1 and TIMP-2 in the progression of cerebral aneurysms," Stroke, vol. 38, no. 8, pp. 2337-2345, 2007.

[74] T. Aoki, H. Kataoka, R. Ishibashi et al., "Pitavastatin suppresses formation and progression of cerebral aneurysms through inhibition of the nuclear factor $\kappa \mathrm{B}$ pathway," Neurosurgery, vol. 64, no. 2, pp. 357-365, 2009.

[75] T. Aoki, H. Kataoka, R. Ishibashi, K. Nozaki, and N. Hashimoto, "Cathepsin B, K, and S are expressed in cerebral aneurysms and promote the progression of cerebral aneurysms," Stroke, vol. 39, no. 9, pp. 2603-2610, 2008.

[76] L. Gu, Y. Okada, S. K. Clinton et al., "Absence of monocyte chemoattractant protein-1 reduces atherosclerosis in low density lipoprotein receptor-deficient mice," Molecular Cell, vol. 2, no. 2, pp. 275-281, 1998.

[77] J. Reckless, E. M. Rubin, J. B. Verstuyft, J. C. Metcalfe, and D. J. Grainger, "Monocyte chemoattractant protein-1 but not tumor necrosis factor- $\alpha$ is correlated with monocyte infiltration in mouse lipid lesions," Circulation, vol. 99, no. 17, pp. 2310-2316, 1999.

[78] C. Shi, I. A. Awad, N. Jafari et al., "Genomics of human intracranial aneurysm wall," Stroke, vol. 40, no. 4, pp. 12521261, 2009.

[79] T. Aoki, H. Kataoka, R. Ishibashi, K. Nozaki, and N. Hashimoto, "Gene expression profile of the intima and media of experimentally induced cerebral aneurysms in rats by laser-microdissection and microarray techniques," International Journal of Molecular Medicine, vol. 22, no. 5, pp. 595-603, 2008.

[80] N. Sadamasa, K. Nozaki, H. Kita-Matsuo et al., "Gene expression during the development of experimentally induced cerebral aneurysms," Journal of Vascular Research, vol. 45, no. 4, pp. 343-349, 2008.

[81] T. Aoki, M. Nishimura, H. Kataoka, R. Ishibashi, K. Nozaki, and N. Hashimoto, "Reactive oxygen species modulate growth of cerebral aneurysms: a study using the free radical scavenger edaravone and $447 \mathrm{phox}^{-1-}$ mice," Laboratory Investigation, vol. 89, no. 7, pp. 730-741, 2009. 
[82] T. Moriwaki, Y. Takagi, N. Sadamasa, T. Aoki, K. Nozaki, and N. Hashimoto, "Impaired progression of cerebral aneurysms in interleukin-1 $\beta$-deficient mice," Stroke, vol. 37, no. 3, pp. 900-905, 2006.

[83] S. Fukuda, N. Hashimoto, H. Naritomi et al., "Prevention of rat cerebral aneurysm formation by inhibition of nitric oxide synthase," Circulation, vol. 101, no. 21, pp. 2532-2538, 2000.

[84] T. Aoki, M. Nishimura, H. Kataoka et al., "Role of angiotensin II type 1 receptor in cerebral aneurysm formation in rats," International Journal of Molecular Medicine, vol. 24, no. 3, pp. 353-359, 2009.

[85] H. Ohkuma, S. Suzuki, S. Fujita, and W. Nakamura, "Role of a decreased expression of the local renin-angiotensin system in the etiology of cerebral aneurysms," Circulation, vol. 108, no. 7, pp. 785-787, 2003.

[86] T. Aoki, M. Nishimura, R. Ishibashi, H. Kataoka, Y. Takagi, and N. Hashimoto, "Toll-like receptor 4 expression during cerebral aneurysm formation: laboratory investigation," Journal of Neurosurgery, vol. 113, no. 4, pp. 851-858, 2010.

[87] T. Aoki, H. Kataoka, M. Shimamura et al., "NF- $\kappa$ B is a key mediator of cerebral aneurysm formation," Circulation, vol. 116, no. 24, pp. 2830-2840, 2007.

[88] T. Aoki, T. Moriwaki, Y. Takagi et al., "The efficacy of apolipoprotein E deficiency in cerebral aneurysm formation," International Journal of Molecular Medicine, vol. 21, no. 4, pp. 453-459, 2008.

[89] N. Sadamasa, K. Nozaki, and N. Hashimoto, "Disruption of gene for inducible nitric oxide synthase reduces progression of cerebral aneurysms," Stroke, vol. 34, no. 12, pp. 2980-2984, 2003.

[90] L. D. Jou, D. H. Lee, H. Morsi, and M. E. Mawad, "Wall shear stress on ruptured and unruptured intracranial aneurysms at the internal carotid artery," American Journal of Neuroradiology, vol. 29, no. 9, pp. 1761-1767, 2008.

[91] A. Mantha, C. Karmonik, G. Benndorf, C. Strother, and R. Metcalfe, "Hemodynamics in a cerebral artery before and after the formation of an aneurysm," American Journal of Neuroradiology, vol. 27, no. 5, pp. 1113-1118, 2006.

[92] H. Nakatani, N. Hashimoto, Y. Kang et al., "Cerebral blood flow patterns at major vessel bifurcations and aneurysms in rats," Journal of Neurosurgery, vol. 74, no. 2, pp. 258-262, 1991.

[93] E. Metaxa, M. Tremmel, S. K. Natarajan et al., "Characterization of critical hemodynamics contributing to aneurysmal remodeling at the basilar terminus in a rabbit model," Stroke, vol. 41, no. 8, pp. 1774-1782, 2010.

[94] L. Gao, Y. Hoi, D. D. Swartz, J. Kolega, A. Siddiqui, and H. Meng, "Nascent aneurysm formation at the basilar terminus induced by hemodynamics," Stroke, vol. 39, no. 7, pp. 20852090, 2008.

[95] L. M. Khachigian, N. Resnick, M. A. Gimbrone Jr., and T. Collins, "Nuclear factor- $\kappa \mathrm{B}$ interacts functionally with the platelet-derived growth factor B-chain shear-stress response element in vascular endothelial cells exposed to fluid shear stress," Journal of Clinical Investigation, vol. 96, no. 2, pp. 1169-1175, 1995.

[96] Z. Wang, J. Kolega, Y. Hoi et al., "Molecular alterations associated with aneurysmal remodeling are localized in the high hemodynamic stress region of a created carotid bifurcation," Neurosurgery, vol. 65, no. 1, pp. 169-177, 2009.

[97] R. Morishita, N. Tomita, Y. Kaneda, and T. Ogihara, "Molecular therapy to inhibit $\mathrm{NF} \kappa \mathrm{B}$ activation by transcription factor decoy oligonucleotides," Current Opinion in Pharmacology, vol. 4, no. 2, pp. 139-146, 2004.
[98] T. Aoki and M. Nishimura, "Targeting chronic inflammation in cerebral aneurysms: focusing on NF- $\kappa \mathrm{B}$ as a putative target of medical therapy," Expert Opinion on Therapeutic Targets, vol. 14, no. 3, pp. 265-273, 2010.

[99] H. Kataoka and T. Aoki, "Molecular basis for the development of intracranial aneurysm," Expert Review of Neurotherapeutics, vol. 10, no. 2, pp. 173-187, 2010.

[100] J. K. Liao and U. Laufs, "Pleiotropic effects of statins," Annual Review of Pharmacology and Toxicology, vol. 45, pp. 89-118, 2005.

[101] N. Kimura, H. Shimizu, H. Eldawoody et al., "Effect of olmesartan and pravastatin on experimental cerebral aneurysms in rats," Brain Research, vol. 1322, pp. 144-152, 2010.

[102] R. Ishibashi, T. Aoki, M. Nishimura, N. Hashimoto, and S. Miyamoto, "Contribution of mast cells to cerebral aneurysm formation," Current Neurovascular Research, vol. 7, no. 2, pp. 113-124, 2010. 


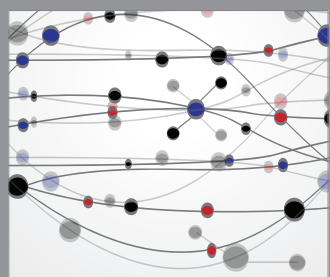

The Scientific World Journal
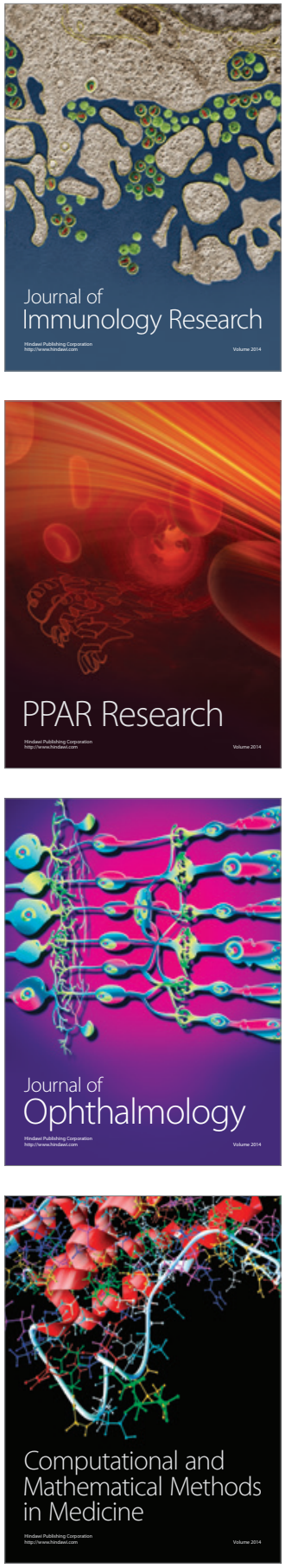

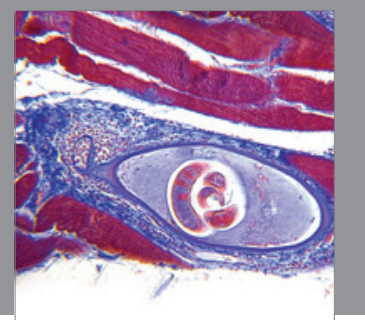

Gastroenterology

Research and Practice
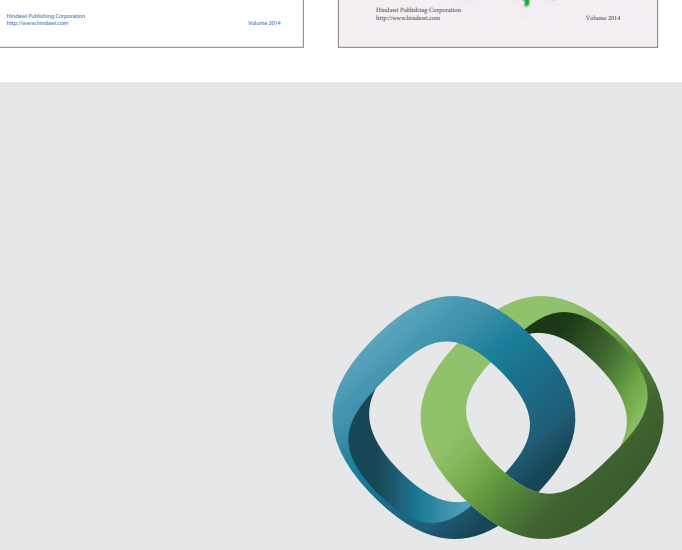

\section{Hindawi}

Submit your manuscripts at

http://www.hindawi.com
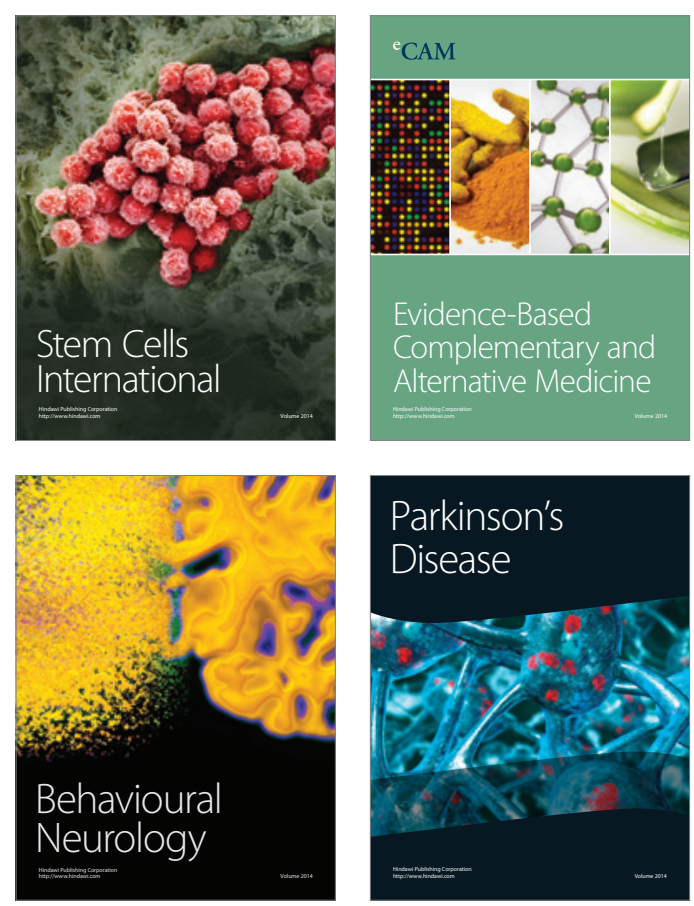

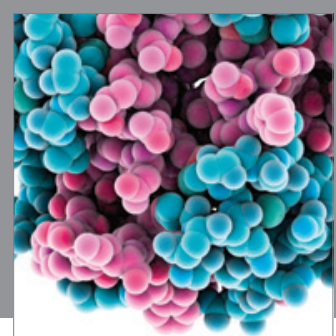

Journal of
Diabetes Research

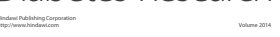

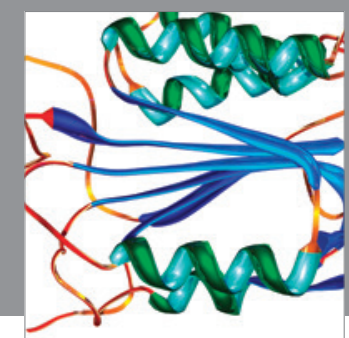

Disease Markers
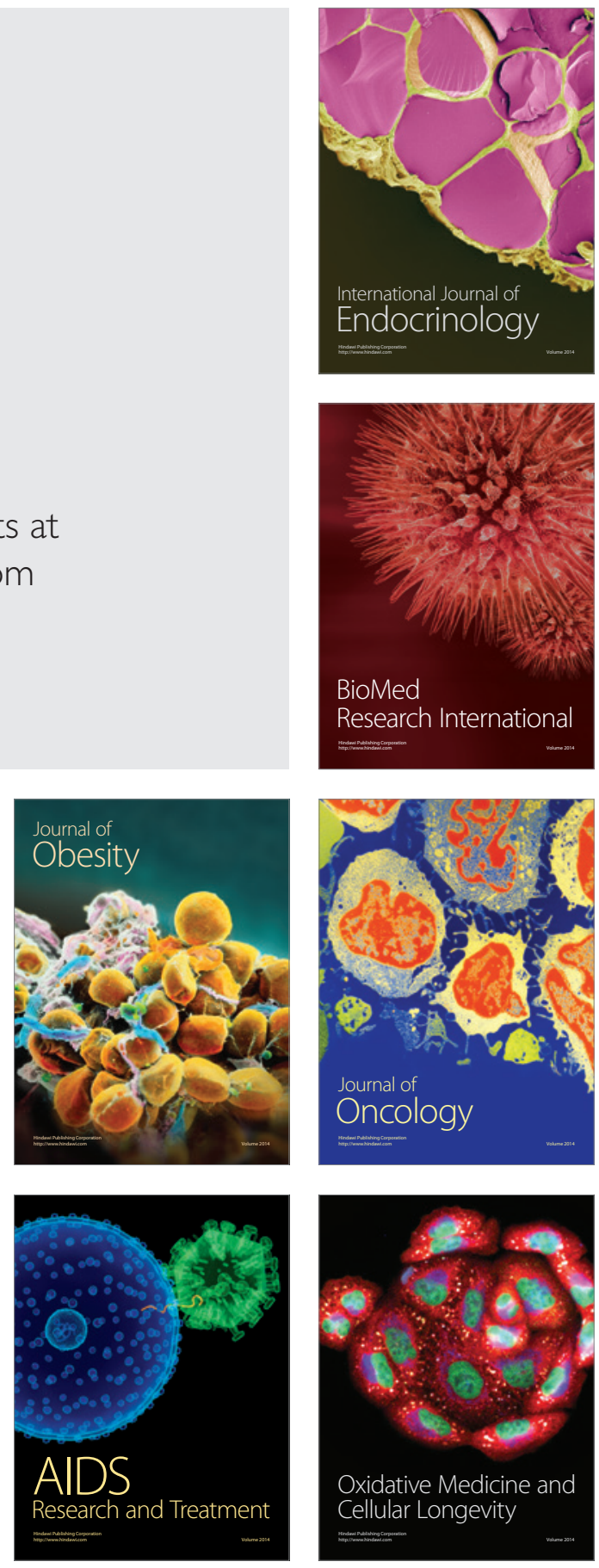